\title{
Strategic interaction in interacting particle systems
}

\author{
Paolo Dai Pra, Elena Sartori and Marco Tolotti
}

\begin{abstract}
In the last decades, models inspired by statistical mechanics have been vastly used in the context of social sciences to model the behavior of interacting economic actors. In particular, parallel updating models such as Probabilistic Cellular Automata have been proved to be very useful to represent rational agents aiming at maximize their utility in the presence of social externalities. What PCA do not account for is strategic interaction, i.e., the fact that, when deciding, agents forecast the action of other agents. In this contribution, we compare models that differ in the presence of strategic interaction and memory of past actions. We will show that the emergent equilibria can be very different: fixed points, cycles of period 2 and chaotic behavior may appear and, possibly, coexist for some values of the parameters of the model.
\end{abstract}

\section{Introduction}

The idea that principles of statistical physics could be applied to systems comprised by a large number of intelligent and rational individuals has fascinated physicists and mathematicians for several decades, besides having stimulated the imagination of science fiction writers. The number of individuals in a reasonably large community, although quite far from the Avogadro number, is large enough to separate microscopic from macroscopic scale; in more technical terms, a reasonably simple collective behavior should result from the combination of all individual behaviors

Paolo Dai Pra

Dipartimento di Matematica, Università di Padova, Padova, Italy, e-mail: daipra@math.unipd.it

Elena Sartori

Dipartimento di Matematica, Università di Padova, Padova, Italy, e-mail: esartori@math.unipd.it

Marco Tolotti

Department of Management, Università Ca' Foscari, Venezia, Italy, e-mail: tolotti@ unive.it 
through a law of large numbers, in analogy to the way thermodynamic quantities such as pressure and temperature result from the combination of the irregular motion of single particles in a gas. Moreover, some stylized features of the interaction between individuals (e.g. imitation) show similarities with interactions between the elements of some physical systems (e.g. spin interactions in ferromagnets).

Together with the analogies mentioned above, many differences naturally emerge. In physical systems, the prime principles on which the microscopic dynamics are based, are usually well established, as they follow from fundamental laws. This is not the case in social systems: interactions between individuals are complex and not well understood. Any stylized model is thus likely to have limited applicability. At a more technical level, some standard assumptions in models inspired by Statistical Physics, such as time reversibility and short range of the interaction, are often unreasonable in the dynamics of social systems.

Our view is that, despite of these difficulties, modeling of large scale social systems is a relevant and stimulating challenge. Stylized models, though unrealistic, may reveal the key factors producing certain behaviors, allowing, for instance, the design of controls to avoid the emergence of undesirable patterns in real social systems. We remark that similar ideas have proved to be successful in other contexts, e.g. biology with applications to medicine (see e.g. [6]).

As we mentioned already, stylized modeling for systems of interacting rational individuals has been vastly inspired by statistical mechanics. After all, elementary particles are themselves "rational", in that they aim at minimizing their contribution to the total energy of the system; temperature injects noise in the dynamics, causing entropic effects to be macroscopically relevant. In this spirit, it has been remarked in several works (see e.g. $[2,1]$ ) that many discrete time stochastic models for the evolution of interacting particles can be formulated as a sequence of optimizations: at each time a particle "chooses" its next position by minimizing its contribution to the energy of the system given the position of all other particles, and subject to some random disturbance. As we will see later, this interpretation is particularly natural in the context of parallel updating of Probabilistic Cellular Automata (PCA). This formulation appears suitable for applications to social sciences, where "minimizing particle's contribution to the total energy of the system" is replaced by "maximizing individual utility". Since individual utilities can be arbitrary functions of positions of all individuals, one obtains a wide class of models, not necessarily time reversible.

The purpose of this paper is to propose a modification of this approach to modeling interacting systems, which takes into account one basic difference between the "rationality" of physical particles and that of human individuals. We, simply, express it as follows: in interacting with other humans, any individual tries to forecast what the others will be doing in the (near) future. It is easy to exhibit examples in which this forecasting plays a relevant role. An obvious example is that of agents investing in a financial market. Each agent aims at maximizing his own profit; this profit depends of the future prices of assets, which in turns depends of the future investment strategy of all agents. Naturally the agent tries to forecast the strategies of other individuals, well aware that all the others will do the same. 
An example in a different context is that of a car traffic jam in an intersection with a broken traffic light. A driver will decide to cross the intersection when he is reasonably confident that drivers coming from other directions will not; his decision is based on what he believes the behavior of other individuals will be. It has been observed (see e.g. [7]) that the evolution rapidly goes to a self-organized steady state, where crossings from concurrent directions alternate in a nearly periodic way. The problem of formulating stylized models exhibiting this behavior is, to a large extent, still unsolved (see [9]).

In this paper, elaborating on ideas contained in [3], we propose a formulation of what we will refer to as strategic interaction, i.e. a mechanism of interaction between individuals which includes forecasting of other individuals' behavior in the near future. Rather than aiming at generality, we illustrate our basic ideas in a very simple context, inspired by PCA's. In Section 2 we illustrate the interpretation of PCA's as sequential stochastic optimization problems, and we propose the version of the same models with strategic interaction. In Section 3 we analyze a simple mean-field model, for which the macroscopic limit is easily obtained, and illustrate the effects of the strategic interaction on the steady state behavior.

\section{Strategic and non-strategic interaction}

By Probabilistic Cellular Automata (PCA) we, generally, mean a discrete time Markov chain on a product space $S^{\Lambda}$, where $\Lambda$ is finite or countable, whose transition probabilities are product measures; in other words, different components update simultaneously and independently. In this paper we restrict to the case in which $S=\{-1,1\}$ and $\Lambda$ is finite. It will be clear that most ideas apply to more general choices of $S$, but binary models allow some peculiar explicit computation. We denote by $\sigma=\left(\sigma_{i}\right)_{i \in \Lambda}$ an element of $\{-1,1\}^{\Lambda}$. The evolution $(\sigma(n))_{n \geq 0}$ of a PCA is of the product form

$$
\mathbb{P}(\sigma(n+1)=\sigma \mid \sigma(n)=\xi)=\prod_{i \in \Lambda} \mathbb{P}\left(\sigma_{i}(n+1)=\sigma_{i} \mid \sigma(n)=\xi\right)=: \prod_{i \in \Lambda} p_{i}\left(\sigma_{i} \mid \xi\right) .
$$

If we assume $p_{i}\left(\sigma_{i} \mid \xi\right)>0$ for every $i, \sigma, \xi$, then $p_{i}\left(\sigma_{i} \mid \xi\right)$ can be written in the form $p_{i}\left(\sigma_{i} \mid \xi\right)=\exp \left[\sigma_{i} \Phi_{i}(\xi)\right] /\left[2 \cosh \left(\Phi_{i}(\xi)\right)\right]$, for some functions $\Phi_{i}:\{-1,1\}^{\Lambda} \rightarrow \mathbb{R}$. Without any loss of generality, for the interpretation of the model it is convenient to introduce some parameters, writing $\Phi_{i}$ in the following form:

$$
\Phi_{i}(\xi)=\beta\left[F_{i}(\xi)+\mu_{i} \xi_{i}\right], \quad \text { for } \beta, \mu_{i} \geq 0,
$$

which corresponds to the local transition probabilities

$$
p_{i}\left(\sigma_{i} \mid \xi\right)=\frac{\exp \left[\beta \sigma_{i}\left[F_{i}(\xi)+\mu_{i} \xi_{i}\right]\right.}{2 \cosh \left(\beta\left[F_{i}(\xi)+\mu_{i} \xi_{i}\right]\right)} .
$$


The function $\Phi$ in (2) can be interpreted as follows.

- $\quad \beta$ is an inverse temperature parameter, that allows to tune the amount of random noise in the system. For $\beta=0$ the system is fully random. As $\beta \rightarrow+\infty$, the dynamics converge to the (nearly) deterministic evolution

$$
\sigma_{i}(n+1)=\operatorname{sign}\left[F_{i}(\sigma(n))+\mu_{i} \sigma_{i}(n)\right]
$$

randomness only survives in the case $F_{i}(\sigma(n))+\mu_{i} \sigma_{i}(n)=0$, where the values \pm 1 for $\sigma_{i}(n+1)$ are equally probable.

- The function $F_{i}$ describes the interaction of the $i$-th component with the others.

- The parameter $\mu_{i}$ models friction: for large $\mu_{i}$ it is unlikely for $\sigma_{i}$ to change sign in a time step, slowing down the dynamics (see [4], where this friction plays a key role for determining a "desired" stationary distribution). Of course, the term $\mu_{i} \xi_{i}$ could be included in $F_{i}(\xi)$, but it will be convenient to separate this selfinteraction term.

\subsection{An equivalent optimization problem}

Suppose each $i \in \Lambda$ labels an agent that, at any time $t$, faces a binary decision problem: $\sigma_{i}(n)= \pm 1$ denotes the action of the $i$-th agent at time $n$. At each time $n$ the aim of the $i$-th agent is to maximize a random utility function $U_{i}$ as function of the action $s_{i}=\sigma_{i}(n)$; the function $U_{i}\left(s_{i}\right)$ is determined by the action of (possibly) all agents at time $n-1$, and by a random term $\varepsilon_{i}(n)$ as follows:

$$
U_{i}\left(s_{i} ; \sigma(n-1), \varepsilon_{i}(n)\right):=s_{i}\left[F_{i}(\sigma(n-1))+\mu_{i} \sigma_{i}(n-1)+\varepsilon_{i}(n)\right],
$$

where $\left(\varepsilon_{i}(n)\right)_{i \in \Lambda, n \geq 1}$ are i.i.d. real random variables, having the following distribution function:

$$
\eta(x):=\mathbb{P}\left(\varepsilon_{i}(n) \leq x\right)=\frac{1}{1+e^{-2 \beta x}} .
$$

All agents perform simultaneously their optimization. Note that agent $i$ will choose $\sigma_{i}(n)=1$ if and only if

$$
F_{i}(\sigma(n-1))+\mu \sigma_{i}(n-1)+\varepsilon_{i}(n)>0
$$

which, given $\sigma(n-1)$, happens with probability

$$
\begin{aligned}
\mathbb{P}\left[F_{i}(\sigma(n-1))+\mu \sigma_{i}(n-1)+\varepsilon_{i}\right. & (n)>0] \\
= & \eta\left(F_{i}(\sigma(n-1))+\mu \sigma_{i}(n-1)\right) \\
= & \frac{\exp \left[\beta\left[F_{i}(\sigma(n-1))+\mu_{i} \sigma_{i}(n-1)\right]\right.}{2 \cosh \left(\beta\left[F_{i}(\sigma(n-1))+\mu_{i} \sigma_{i}(n-1)\right]\right)} .
\end{aligned}
$$


Note that the case in which equality holds in (6), which would make the two actions equivalent, can be ignored, since it occurs with probability zero.

Comparing (7) with (3), we realize the sequence of optimization problems induces a Markov evolution with the same transition probabilities as the PCA in (3).

\subsection{Strategic interaction}

In the sequence of optimization problems described above, agents update their action simultaneously and independently, on the basis of the past actions. We consider a modification of the model, suggested by the following considerations.

a) The utility of agent $i$ at time $n$ may depend on the action of all agents at the same time $n$.

b) Each agent is aware of the fact that all other agents are optimizing their own utility, and uses this fact to forecast their actions.

c) Agents know all function $F_{i}$ and friction parameters $\mu_{i}$. The random term $\varepsilon_{i}(n)$ can only be observed by agent $i$. Agents know the distribution of $\varepsilon_{i}(n)$.

By c), the action $s_{i}=\sigma_{i}(n)$ of agent $i$ at time $n$ may depend on $\varepsilon_{i}(n)$, so it is convenient to define an action $s_{i}$ as a measurable function $s_{i}\left(\varepsilon_{i}\right)$ of the random term $\varepsilon_{i}$.

If $s=\left(s_{i}\right)_{i \in \Lambda}$ denotes the vector of actions at a given time $n$, we set $s^{i}=\left(s_{j}\right)_{j \neq i}$. We assume agent $i$ aims at maximizing in $s_{i}$ the following utility, obtained modifying in a simple way (4):

$$
U_{i}\left(s_{i}, s^{i} ; \sigma(n-1), \varepsilon_{i}(n)\right)=s_{i}\left[\mathbb{E}^{i}\left(F_{i}(s)\right)+\mu_{i} \sigma_{i}(n-1)+\varepsilon_{i}(n)\right],
$$

where, for a vector of actions $\left(s_{j}\left(\varepsilon_{j}(n)\right)\right)_{j \in \Lambda}=: s(\varepsilon(n))$, the expression $\mathbb{E}^{i}\left(F_{i}(s)\right)$ is obtained by averaging $F_{i}(s(\varepsilon(n)))$ over $\left(\varepsilon_{j}(n)\right)_{j \neq i}$.

Unlike in (4), the utility $U_{i}$ depends on the action of all agents; it is, therefore, natural to give a game-theoretic definition of an "optimal" vector of actions.

Definition 1. A vector of actions $s=\left(s_{i}\left(\varepsilon_{i}(n)\right)\right)_{i \in \Lambda}$ is called a Nash equilibrium if for all $i \in \Lambda$

$$
s_{i}\left(\varepsilon_{i}(n)\right)=\operatorname{argmax} U_{i}\left(\cdot, s^{i} ; \sigma(n-1), \varepsilon_{i}(n)\right) .
$$

In other words, in a Nash equilibrium any agent is using the best action given the other agents' actions. For comments and details on this notion of equilibrium we refer the reader to [8]. It is immediate, but quite relevant, to observe that $s$ is a Nash equilibrium if and only if it is a fixed point for the so-called best response map $s \mapsto \Phi(s)$ given by

$$
\Phi_{i}(s)=\operatorname{argmax} U_{i}\left(\cdot, s^{i} ; \sigma(n-1), \varepsilon_{i}(n)\right) .
$$

In general, in games there is no guarantee that either existence or uniqueness hold for Nash equilibria. For the models above, existence is not a problem, however. 
Proposition 1. At least one Nash equilibrium exists.

Proof. By (10), $\Phi_{i}(s)\left(\varepsilon_{i}(n)\right)$ is increasing in $\varepsilon_{i}(n)$. Thus we can restrict to actions $s$ of the form

$$
s_{i}\left(\varepsilon_{i}\right)=\mathbf{1}_{\left(x_{i},+\infty\right)}\left(\varepsilon_{i}\right),
$$

with $x_{i} \in \overline{\mathbb{R}}:=\mathbb{R} \cup\{ \pm \infty\}$. Thus $\Phi$ can be seen as a map from $\overline{\mathbb{R}}^{|\Lambda|}$ to itself. The fact that the distribution of the $\varepsilon_{i}(n)$ is absolutely continuous guarantees that this map is continuous. Since $\overline{\mathbb{R}}^{|\Lambda|}$ is convex and compact, the conclusion follows from a standard fixed point argument.

The uniqueness of the Nash equilibrium is, however, not guaranteed. This means that, the map which to $(\sigma(n-1), \varepsilon(n))$ associates $\sigma(n)$ may be not single-valued. In order to obtain well-defined Markovian dynamics, one should have a rule for selecting one specific Nash equilibrium; or, otherwise, one should content himself for having defined just a set of possible evolutions of the system. This point will be discussed in more details in a specific model, in Section 3.

\subsection{Trend-driven dynamics}

In many applications the utility of an agent can be interpreted as the return of an investment. This return is determined by the variation of the value of an asset which, in turn, depends of the variation of demand for the asset. To model this situation it is reasonable to assume that the utility $U_{i}$ of agent $i$ depends of the variation (trend) of a function $F_{i}(\sigma)$ of all actions. In the strategic case, which is the most meaningful in this interpretation, this amounts to define the following utility:

$$
U_{i}\left(s_{i}, s^{i} ; \sigma(n-1), \varepsilon_{i}(n)\right)=s_{i}\left[\mathbb{E}^{i}\left[F_{i}(s)\right]-F_{i}(\sigma(n-1))+\mu_{i} \sigma_{i}(n-1)+\varepsilon_{i}(n)\right]
$$

We remark that in absence of friction ( $\mu_{i}=0$ for all $i \in \Lambda$ ), the utility (8) does not depend on $\sigma(n-1)$, so that the resulting evolution $(\sigma(n))_{n \geq 0}$ is a possibly multiplevalued i.i.d. sequence. In the case of utility (11) this is not the case. Clearly, one could consider further generalizations in which a trend term is added to utility (8).

Note that this dynamics driven by the trend can be adapted to the non-strategic context, by letting

$$
\begin{aligned}
U_{i}\left(s_{i} ; \sigma(n-1), \sigma(n-2), \varepsilon_{i}(n)\right) \\
:=s_{i}\left[F_{i}(\sigma(n-1))-F_{i}(\sigma(n-2))+\mu_{i} \sigma_{i}(n-1)+\varepsilon_{i}(n)\right],
\end{aligned}
$$

Rather than aiming at generality, we study some specific simple models, for which the thermodynamic limit $(|\Lambda| \rightarrow+\infty)$ can be obtained explicitly, and compare the long time behaviors of such models in the strategic and non-strategic case. 


\section{A linear, mean-field model}

In this section we consider an homogeneous, mean-field model, for which $\Lambda=$ $\{1,2, \ldots, N\}, \mu_{i} \equiv \mu \geq 0$, and

$$
F_{i}(s)=k m_{N}(s)
$$

where $k \geq 0$ and

$$
m_{N}(s):=\frac{1}{N} \sum_{i=1}^{N} s_{i} .
$$

Our aim is to analyze the $N \rightarrow+\infty$ limit of the random dynamics produced by the sequence of utility optimizations. We will consider all versions (4), (8), (11), (12) of the utility. We begin by briefly treating the case of non-strategic optimization, in order to better appreciate the effects of the game-theoretic setting.

\subsection{The non-strategic case}

We consider first the utility (4). In this case we obtain the stochastic dynamics

$$
\sigma_{i}(n)=\operatorname{sign}\left[k m_{N}(\sigma(n-1))+\mu \sigma_{i}(n-1)+\varepsilon_{i}(n)\right]
$$

Given a set $A$ and $x \in A^{N}$, we introduce the empirical measure

$$
\rho_{N}^{x}:=\frac{1}{N} \sum_{i=1}^{N} \delta_{x_{i}}
$$

Thus, for instance,

$$
\rho_{N}^{\sigma(n)}=\frac{1+m_{N}(\sigma(n))}{2} \delta_{1}+\frac{1-m_{N}(\sigma(n))}{2} \delta_{-1} .
$$

while $\rho_{N}^{\sigma(n-1), \varepsilon(n)}$ is the joint empirical measure of $(\sigma(n-1), \varepsilon(n))$. Averaging over $n$ equation (2) we obtain

$$
m_{N}(\sigma(n))=\int \operatorname{sign}\left[k m_{N}(\sigma(n-1))+\mu s+e\right] \rho_{N}^{\sigma(n-1), \varepsilon(n)}(d s, d e) .
$$

For each $N$, we fix a deterministic initial condition $\sigma(0)$ such that the following limit exists:

$$
\lim _{N \rightarrow+\infty} m_{N}(\sigma(0))=: m(0) .
$$

Then one can prove by induction the following law of large numbers.

Proposition 2. For every $h:\{-1,1\} \times \mathbb{R}$ bounded and continuous 


$$
\begin{aligned}
& \lim _{N \rightarrow+\infty} \int h(s, e) \rho_{N}^{\sigma(n-1), \varepsilon(n)}(d s, d e) \\
& \quad=\int h(s, e)\left(\frac{1+m(n-1)}{2} \delta_{1}+\frac{1-m(n-1)}{2} \delta_{-1}\right)(d s) d \eta(e),
\end{aligned}
$$

where $(m(n))_{n \geq 0}$ solves the recursion

$$
\begin{aligned}
m(n)= & (1+m(n-1)) \eta(k m(n-1)+\mu) \\
& +(1-m(n-1)) \eta(k m(n-1)-\mu)-1=: G_{1}(m(n-1)),
\end{aligned}
$$

with initial condition $m(0)$. Moreover, for each $n \geq 1, m_{N}(\sigma(n))$ converges in probability to $m(n)$, as $N \rightarrow+\infty$

Equation (5) describes the macroscopic dynamics of the system with a large number of agents. The long time behavior of these dynamics is obtained by studying the steady state solutions of (5).

Proposition 3. 1. Assume $\beta k \leq\left(1+e^{-2 \beta \mu}\right) / 2$. Then $\bar{m}=0$ is the unique fixed point for (5). Moreover it is a global attractor, i.e. for every $m(0) \in[-1,1]$

$$
\lim _{n \rightarrow+\infty} m(n)=0 .
$$

2. For $\beta k>\left(1+e^{-2 \beta \mu}\right) / 2, \bar{m}=0$ is an unstable fixed point. Moreover there is $m^{*}>$ 0 such that $\pm m^{*}$ are locally stable fixed points and, for every $m(0) \in[-1,1] \backslash\{0\}$

$$
\lim _{n \rightarrow+\infty} m(n) \in\left\{-m^{*}, m^{*}\right\} .
$$

Thus (5) has the familiar behavior of the Curie-Weiss model. In comparison with the standard Curie-Weiss model, a slight difficulty is due to the fact that the function $G_{1}(\cdot)$ in (5) is non necessarily concave in $[0,1]$; one however shows that $G_{1}(\cdot)$ is actually concave for $\beta k \leq \frac{1+e^{-2 \beta \mu}}{2}$, and at most one change of concavity occurs otherwise. With this remark the standard proof for the Curie-Weiss model is easily adapted. Details are omitted here.

Let us now turn to the trend-dependent utility (11). The above argument for the derivation of the macroscopic dynamics can be repeated; equation (5) is now replaced by

$$
\begin{aligned}
m(n)= & (1+m(n-1)) \eta(k[m(n-1)-m(n-2)]+\mu) \\
& +(1-m(n-1)) \eta(k[m(n-1)-m(n-2)]-\mu)-1,
\end{aligned}
$$

where we need to assume $\left(m_{N}(0), m_{N}(1)\right) \rightarrow(m(0), m(1))$. The analysis of the steady states for (6) is considerably harder. Although not yet fully proved, the following picture is supported by strong numerical evidence (see Fig. 3.1).

Conjecture.

Case $\mu=0$. For $\beta k \leq 1, \bar{m}=0$ is a globally attracting fixed point: for every $(m(0), m(1)) \in[-1,1]^{2}$,

$$
\lim _{n \rightarrow+\infty} m(n)=0 .
$$




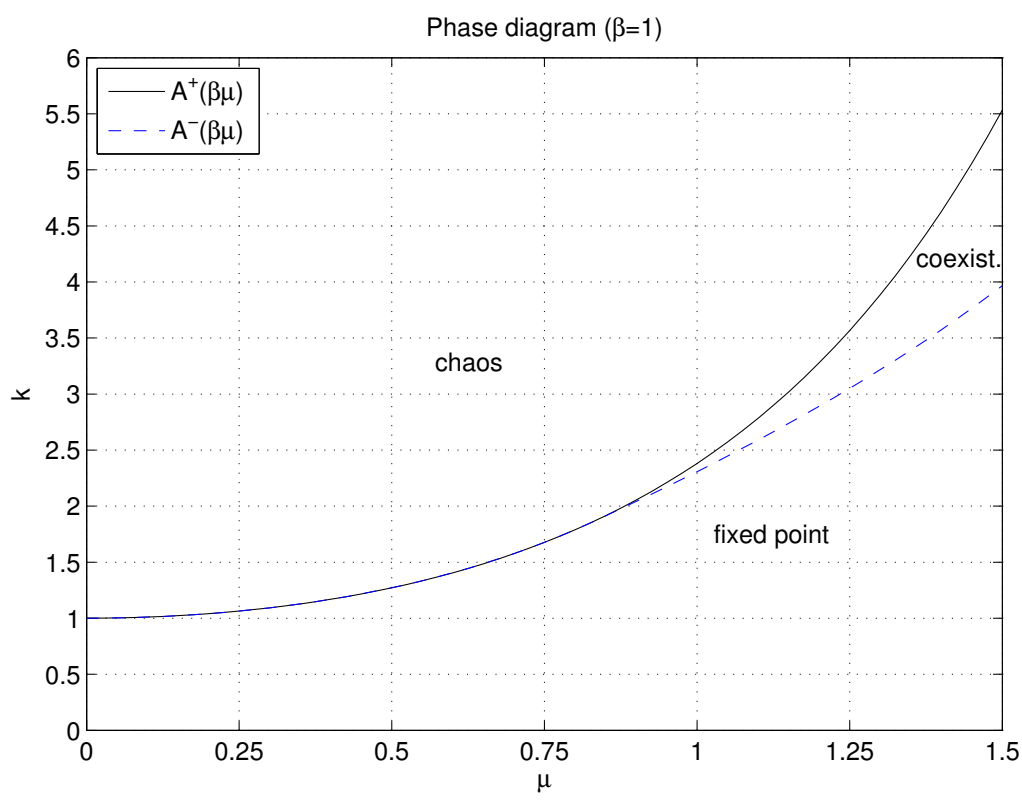

Fig. 1 Phase diagram of steady states.

For $\beta k>1$ all initial conditions, except $(m(0), m(1))=(0,0)$, converge to a periodic trajectory of period 6 of the form $\left(\ldots, x^{*}, x^{*}, 0,-x^{*},-x^{*}, 0, \ldots\right)$ for a unique $x^{*}>0$. Case $\mu>0$. There is a constant $A^{-}(\beta \mu) \leq A^{+}(\beta \mu):=[\cosh (\beta \mu)+1] /(2 \beta)$ such that:

- for $\beta k \leq A^{-}(\beta \mu)$ all initial conditions are attracted to zero;

- for $\beta k \geq A^{+}(\beta \mu)$ for no initial condition, except $(m(0), m(1))=(0,0)$, the trajectory converges to 0 ;

- for $A^{-}(\beta \mu)<\beta k<A^{+}(\beta \mu)$, we have that $m(n) \rightarrow 0$ if and only if $(m(0), m(1)) \in$ $\mathscr{N}$, where $\mathscr{N} \subsetneq[-1,1]^{2}$ is a neighborhood of $(0,0)$.

Finally, there is a constant $c>0$ such that $A^{-}(\beta \mu)<A^{+}(\beta \mu)$ if and only if $\beta \mu>c$. In the case $\mu>0$, the trajectories that do not converge to zero appear to have a much more complex behavior. The long time behavior is sensitive to the initial condition, and there is no evidence of locally stable periodic orbits. We observe that, for $\beta \mu>c$ and $A^{-}(\beta \mu)<\beta k<A^{+}(\beta \mu)$, the locally stable fixed point coexists with the "chaotic" phase. 


\subsection{The strategic case: the utility (8)}

In analogy with (2), we begin the analysis by the optimality condition for the utility (8):

$$
\sigma_{i}(n)=\operatorname{sign}\left[k \mathbb{E}^{i}\left[m_{N}(\sigma(n))\right]+\mu \sigma_{i}(n-1)+\varepsilon_{i}(n)\right] .
$$

Equation (7) does not uniquely identify the dynamics, due to the possible multiplicity of Nash equilibria. Nevertheless the following facts hold.

Proposition 4. Assume that, for each $n \geq 1$, it is selected a Nash equilibrium, i.e. a solution of (7). Then the resulting stochastic process $\left(m_{N}(\sigma(n))\right)_{n \geq 0}$ is tight, and each weak limit point satisfies a.s. the implicit equation

$$
\begin{aligned}
m(n) & =(1+m(n-1)) \eta(k m(n)+\mu)+(1-m(n-1)) \eta(k m(n)-\mu)-1 \\
& =: G_{2}(m(n), m(n-1)) .
\end{aligned}
$$

For the proof of this result see [3].

The dynamics are now described by the implicit recursion (8). By a standard fixed point argument, it is easily shown that, for every $x \in[-1,1]$, the equation $y=G_{2}(y, x)$ has at least one solution in $[-1,1]$. This is the macroscopic version of the existence of a Nash equilibrium (see Proposition 1). On the other hand, possible multiplicity of Nash equilibria translates to multiple solutions of the equation $y=G_{2}(y, x)$, producing a large set of possible dynamics, i.e. sequences $(m(n))_{n \geq 0}$ satisfying (8). For the study of these dynamics, it is useful to notice that, since $G_{2}(y, x)$ is linear in $x$, the equation $y=G_{2}(y, x)$ determines a function $x=\psi(y)$. Computing the derivative $\psi^{\prime}$, one shows that for $\beta k \leq 1$ the function $\psi$ is strictly increasing on $\mathbb{R}$, and thus it admits an inverse $\phi=\psi^{-1}$; moreover, $\phi$ maps $[-1,1]$ into $[-1,1]$. This simple computation leads to the following result, whose proof is omitted.

Proposition 5. For $\beta k \leq 1$ and for any $\mu>0$, equation (8) gives rise to a singlevalued dynamics.

1. For $\beta k \leq\left(1+e^{-2 \beta \mu}\right) / 2, \bar{m}=0$ is a globally attracting fixed point.

2. For $\left(1+e^{-2 \beta \mu}\right) / 2<\beta k \leq 1, \bar{m}=0$ is an unstable fixed point. Moreover, there is $m^{*}>0$ such that $\pm m^{*}$ are locally stable fixed points and, for every $m(0) \in$ $[-1,1] \backslash\{0\}$

$$
\lim _{n \rightarrow+\infty} m(n) \in\left\{-m^{*}, m^{*}\right\}
$$

Note that the high temperature regime $\beta k \leq\left(1+e^{-2 \beta \mu}\right) / 2$ coincides with that of the corresponding non-strategic model seen in Proposition 3. Moreover, as the threshold $\left(1+e^{-2 \beta \mu}\right) / 2$ is crossed, but $\beta k \leq 1$, the systems enters a polarized phase, again as in the non-strategic model.

But, what happens for $\beta k>1$ ? It is not hard to show that, as $\beta k>1$, if $\mu$ is small enough, the function $\psi$ is not monotonic, and, therefore, it cannot be globally inverted: equation (8) determines multi-valued dynamics. A partial description of these dynamics can be inferred by the following remarks (see also Fig. 3.2). 
a) For all values of the parameters the equation $y=G_{2}(y, x)$ determines a function $y=\phi(x)$ mapping a neighborhood of the origin onto a neighborhood of the origin. This map is contractive, i.e. it has a Lipschitz constant $L<1$ for $\beta k<\left(1+e^{-2 \beta \mu}\right) / 2$ and for $\beta k>\left(1+e^{2 \beta \mu}\right) / 2$. Thus, there is a low temperature regime in which, if $m(0)$ is sufficiently close to 0 , then $m(n) \rightarrow 0$ as $n \rightarrow+\infty$.

b) For all $\beta k>\left(1+e^{2 \beta \mu}\right) / 2$ the equation $x=G_{2}(x, x)$ has exactly three solutions $-m^{*}, 0, m^{*}$, with $m^{*}>0$. Moreover, the equation $y=G_{2}(y, x)$ determines a contractive function $y=\phi(x)$ around $\pm m^{*}$. Thus $\pm m^{*}$ can be seen as locally stable fixed points for the dynamics. Note that, however, for $x$ close to $\pm m^{*}$, the equation $y=G_{2}(y, x)$ does not have necessarily a unique solution.

Unlike the non-strategic case, for large values of $\beta k$ other steady state solutions emerge.

c) For $\beta k$ sufficiently large, depending on the value of $\beta \mu$, the equation $x=$ $G_{2}(x,-x)$ has five solutions $-x^{(2)},-x^{(1)}, 0, x^{(1)}, x^{(2)}$, with $0<x^{(1)}<x^{(2)}$. This means that the trajectories alternating $x^{(1)}$ and $-x^{(1)}$ (or $x^{(2)}$ and $\left.-x^{(2)}\right)$ solve (8), so they are possible dynamics for the system. Around each of these points the equation $y=G_{2}(y, x)$ locally determines a function $y=\phi(x)$; the aforementioned 2 -cycle (trajectory of period two) $\left(-x^{(1)}, x^{(1)}\right)$ is locally stable (whenever it exists) for the dynamics determined by $\phi$, while the 2 -cycle $\left(-x^{(2)}, x^{(2)}\right)$ is locally stable only for $\beta k$ large.

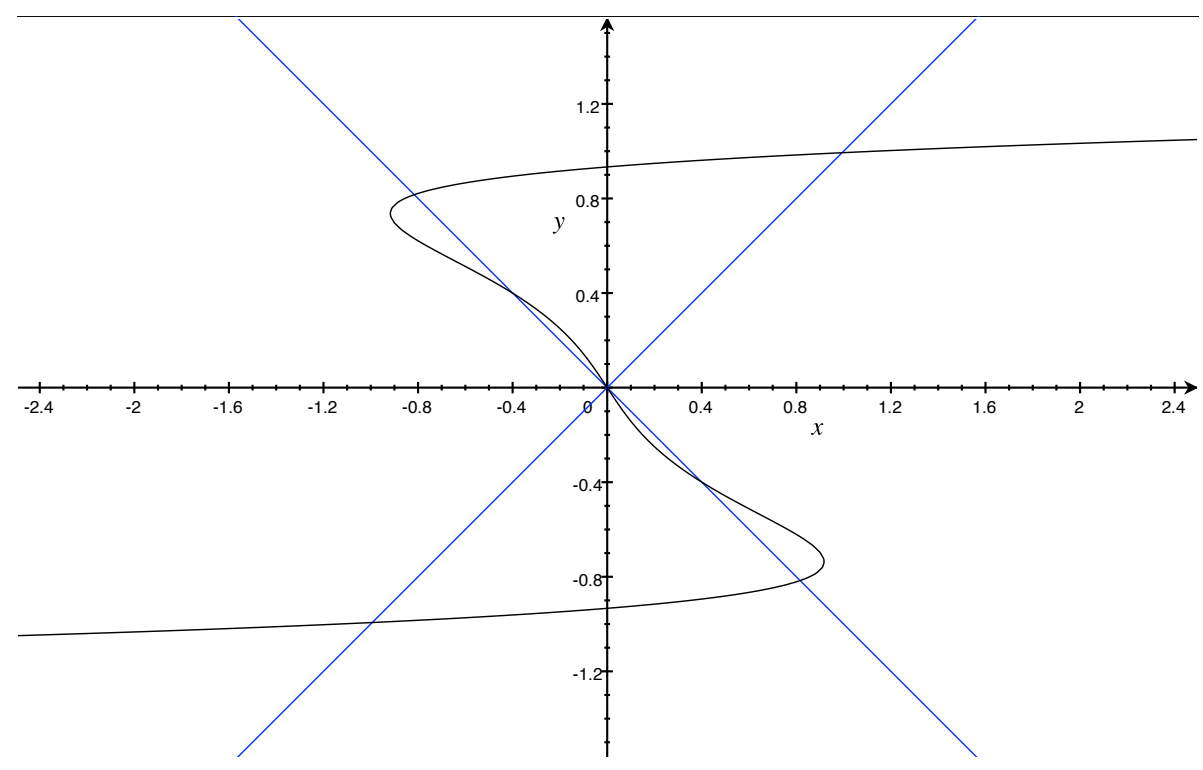

Fig. 2 Graph of the curve $y=G_{2}(y, x)$. Intersections with $y=x$ correspond to fixed points, intersection with $y=-x$ correspond to 2 -cycles. Here the parameters are $\beta=1, \mu=0.7, k=2.2$. 
The fact that the recursion (8) is multi-valued, allows many other possible dynamics. For certain values of the parameters, the system could, for instance, shift from a neighborhood of a fixed point to a neighborhood of a 2-cycle. The selection of one specific dynamics depends on the selection of a particular Nash equilibrium, which corresponds, in the thermodynamic limit, to the choice of a particular solution of $y=G_{2}(y, x)$.

\subsubsection{Selection of Nash equilibria}

Nash equilibria are fixed points for the best response map (10). In the limit of infinitely many agents, these fixed points become, given $m(n-1)$, fixed points for the map $y \mapsto G_{2}(y, m(n-1))$. One possible criterion for the selection of the fixed point, is to assume that a Nash equilibrium is not instantaneously achieved, but it emerges as a result of a learning mechanism, known under the name of Cournot adjustment (see e.g. [5]). Although there are several versions of this mechanism, we only consider it in the simplest version. Assume the dynamics (9), or (8) in the thermodynamic limit, takes place in a discrete time that we call macro-time. Between two successive macro-times $n-1$ and $n$ the following learning mechanism evolves on a fast micro-time: for a given choice of $\xi(0) \in\{-1,1\}^{n}$ we set recursively

$$
\xi_{i}(k+1):=\operatorname{argmax}\left[U_{i}\left(\cdot, \xi^{i}(k) ; \sigma(n-1), \varepsilon_{i}(n)\right)\right],
$$

where $U_{i}$ is given in (8). Fixed points for this micro-dynamics are, by definition, Nash equilibria. In the limit of infinitely many agents, $y_{k}:=\frac{1}{N} \sum_{i} \xi_{i}(k)$ converges to the solution of

$$
y_{k+1}=G_{2}\left(y_{k}, m(n-1)\right) .
$$

Since the map $y \mapsto G_{2}(y, m(n-1))$ is continuous and increasing, the $\operatorname{limit} \lim _{k} y_{k}$ exists, and it is a fixed point. This allows to select a single solution to the equation $y=G_{2}(y, m(n-1))$. The selected solution may depend on the choice of $y_{0}$. This virtually rules out those fixed points $y=G_{2}(y, m(n-1))$ that are unstable for the recursion (9); these points cannot indeed be obtained as limits in the above recursion, unless one chooses $y_{0}=y$. To obtain a single-valued dynamics, one "natural" choice could be to set $y_{0}:=m(n-1)$.

We, now, briefly discuss the implications of this learning procedure. Consider, in particular, the 2 -cycles discussed above. Suppose $-x^{(2)},-x^{(1)}, 0, x^{(1)}, x^{(2)}$, with $0<x^{(1)}<x^{(2)}$, are the solutions of $x=G_{2}(x,-x)$.

(a) Set, to begin with, $m(n-1)=-x^{(1)}$. It can be shown that $y=x^{(1)}$ is an unstable fixed point for the map $y \mapsto G_{2}\left(y,-x^{(1)}\right)$ : for no choice of $y_{0}$ the Cournot adjustment procedure can select the 2-cycle that alternates $-x^{(1)}$ and $x^{(1)}$.

(b)Set now $m(n-1)=-x^{(2)}$. It can be shown that $y=x^{(2)}$ is a locally stable fixed point for the map $y \mapsto G_{2}\left(y,-x^{(2)}\right)$. However, $y_{0}=-x^{(2)}$ is not in its basin of attraction.

Summing up, the following result can be proved. 
Proposition 6. Assume a single-valued dynamics is selected via the Cournot adjustment procedure, with $y_{0}=m(n-1)$. Then, whenever $\beta k>\left(1+e^{-2 \beta \mu}\right) / 2$ and $m(0) \neq 0$, we have

$$
\lim _{n \rightarrow+\infty} m(n) \in\left\{-m^{*}, m^{*}\right\},
$$

where $m^{*}$ is the unique strictly positive solution of $x=G_{2}(x, x)$.

Thus, following the Cournot adjustment procedure with $y_{0}=m(n-1)$, we obtain the same behavior as the corresponding non-strategic model. Stable 2-cycles may exist, indeed coexist with stable fixed points, but require different selection procedures.

\subsection{The strategic case: the utility (12)}

Finally, we are left with the trend-driven, strategic case, whose condition for optimality is given by

$$
\sigma_{i}(n)=\operatorname{sign}\left[k \mathbb{E}^{i}\left[m_{N}(\sigma(n))\right]-k m_{N}(\sigma(n-1))+\mu \sigma_{i}(n-1)+\varepsilon_{i}(n)\right] .
$$

As for Proposition 4, we can obtain the following macroscopic description of the dynamics.

Proposition 7. Assume that, for each $n \geq 1$, it is selected a Nash equilibrium, i.e. a solution of (7). Then the resulting stochastic process $\left(m_{N}(\sigma(n))\right)_{n \geq 0}$ is tight, and each weak limit point satisfies a.s. the implicit equation

$$
\begin{aligned}
m(n)= & (1+m(n-1)) \eta(k m(n)-k m(n-1)+\mu) \\
& +(1-m(n-1)) \eta(k m(n)-k m(n-1)-\mu)-1 \\
= & : G_{3}(m(n), m(n-1)) .
\end{aligned}
$$

The analysis of the steady state solutions of (11) has been done in [3].

Proposition 8. There is a constant $A(\beta \mu) \leq \frac{1+e^{2 \beta \mu}}{4}$ such that:

1. the equation $y=G_{3}(y, x)$ uniquely determines a function $y=\psi(x)$ around $(x, y)=(0,0)$. Moreover $\bar{m}=0$ is stable for the resulting local dynamics if and only if $\beta k \leq\left(1+e^{2 \beta \mu}\right) / 4$;

2. for $\beta k>A(\beta \mu)$ a 2-cycle exists, i.e. a strictly positive solution of $x=G_{3}(x,-x)$. For at least one 2-cycle the dynamics can be locally made explicit, and the 2cycle is stable for this local dynamics;

3. there is a constant $0<c \leq(1 / 2) \log 2$ such that, if $\beta \mu>c$, then $A(\beta \mu)<(1+$ $\left.e^{2 \beta \mu}\right) / 4$. In this case stable fixed points coexist with a stable 2-cycle.

Unlike the model corresponding to utility (8), in this case the analysis of the best response map reveals that 2-cycles survive the Cournot adjustment selection. Partial proofs and numerical simulations strongly support the following conjecture. 
Conjecture. Assume the Cournot adjustment selection with $y_{0}=m(n-1)$ is used to obtain a single-valued dynamics $(m(n))_{n \geq 0}$. There is a constant $A(\beta \mu) \leq(1+$ $\left.e^{2 \beta \mu}\right) / 4$ with the properties stated in Proposition 8 such that:

1. if $\beta k \leq A(\beta \mu)$, then

$$
\lim _{n \rightarrow+\infty} m(n)=0
$$

for every choice of $m(0)$;

2. if $\beta k>\left(1+e^{2 \beta \mu}\right) / 4$ then the trajectory $m(n)$ converges, as $n \rightarrow+\infty$, to a unique stable 2 -cycle, for every $m(0) \neq 0$;

3. if $A(\beta \mu)<\beta k<\left(1+e^{2 \beta \mu}\right) / 4$, then there are $0<\xi^{(1)}<\xi^{(2)}$ such that

i) the trajectory alternating $-\xi^{(1)}$ and $\xi^{(1)}$ is an unstable 2-cycle;

ii) the trajectory alternating $-\xi^{(2)}$ and $\xi^{(2)}$ is a locally stable 2-cycle;

iii)if $|m(0)|<\xi^{(1)}$ then $m(n)$ converges to 0 as $n \rightarrow+\infty$, while, if $|m(0)|>\xi^{(1)}$, then $m(n)$ converges to the stable 2-cycle.

Note that, even with the Cournot adjustment selection, the steady state behavior deeply differs from the one of the corresponding non-strategic model.

\section{Conclusion}

We have introduced a modeling framework to include forecasting in the dynamics of interacting systems and discussed the problem of selecting a Nash equilibrium, making the resulting dynamics single-valued. In a simple mean-field spin model driven by the trend, the introduction of such forecasting dramatically changes the low temperature dynamics, producing organized stable periodic behavior.

Acknowledgments. We thank Marco LiCalzi and Paolo Pellizzari for inspiring discussions.

\section{References}

1. Bouchaud, J.P.: Crises and collective socio-economic phenomena: simple models and challenges. Journal of Statistical Physics 151, 567-606, (2013)

2. Brock, W.A., Durlauf, S.N.: Discrete choice with social interactions. Review of Economic Studies 68, 235-260, (2001)

3. Dai Pra, P., Sartori, E., Tolotti, M.: Strategic interaction in trend-driven dynamics. Journal of Statistical Physics 152, 724-741, (2013)

4. Dai Pra, P., Scoppola, B., Scoppola, E.: Sampling from a Gibbs measure with pair interaction by means of PCA. Journal of Statistical Physics 149, 722-737, (2012)

5. Fudenberg, D. (1998) The theory of learning in games (Vol. 2). MIT press.

6. Landman, K.A., Binder, B.J., Newgreen, D.F (2012) Modeling Development and Disease in Our "Second" Brain. In Cellular Automata (pp. 405-414). Springer Berlin Heidelberg. 
7. Lämmer, S., Helbing, D.: Self-control of traffic lights and vehicle flows in urban road networks. Journal of Statistical Mechanics: Theory and Experiment 2008, P04019, (2012)

8. Myerson, R. B. (2013) Game theory. Harvard university press.

9. Piccoli, B., Tosin, A.: Time-evolving measures and macroscopic modeling of pedestrian flow. Archive for Rational Mechanics and Analysis 199, 707-738, (2011) 